\title{
Investigation of Transonic Wake Dynamics for Mechanically Deployable Entry Systems
}

\author{
Eric Stern \\ University of Minnesota \\ Minneapolis, MN 55455 \\ 612-626-0019 \\ stern177@umn.edu
}

\author{
Michael Barnhardt \\ ERC Inc. \\ MS 230-3 \\ Moffett Field, CA 94035 \\ 650-604-0843
}

Michael.D.Barnhardt@nasa.gov

\author{
Ethiraj Venkatapathy \\ NASA Ames Research Center \\ MS 233-3 \\ Moffett Field, CA 94035 \\ 650-604-4282 \\ Ethiraj.Venkatapathy@nasa.gov
}

\author{
Graham Candler \\ University of Minnesota \\ Minneapolis, MN 55455 \\ 612-625-2364 \\ candler@aem.umn.edu
}

\author{
Dinesh Prabhu \\ ERC Inc. \\ MS-230-3 \\ Moffet Field, CA 94035 \\ 650-604-1145
}

dinesh.k.prabhu@nasa.gov

\begin{abstract}
A numerical investigation of transonic flow around a mechanically deployable entry system being considered for a robotic mission to Venus has been performed, and preliminary results are reported. The flow around a conceptual representation of the vehicle geometry was simulated at discrete points along a ballistic trajectory using Detached Eddy Simulation (DES). The trajectory points selected span the low supersonic to transonic regimes with freestream Mach numbers from 1.5 to 0.8 , and freestream Reynolds numbers (based on diameter) between $2.09 \times 10^{6}$ and $2.93 \times 10^{6}$. Additionally, the Mach 0.8 case was simulated at angles of attack between $0^{\circ}$ and $5^{\circ}$. Static aerodynamic coefficients obtained from the data show qualitative agreement with data from $70^{\circ}$ sphere-cone wind tunnel tests performed for the Viking program. Finally, the effect of choices of models and numerical algorithms is addressed by comparing the DES results to those using a Reynolds Averaged Navier-Stokes (RANS) model, as well as to results using a more dissipative numerical scheme.
\end{abstract}

\section{Table of Contents}

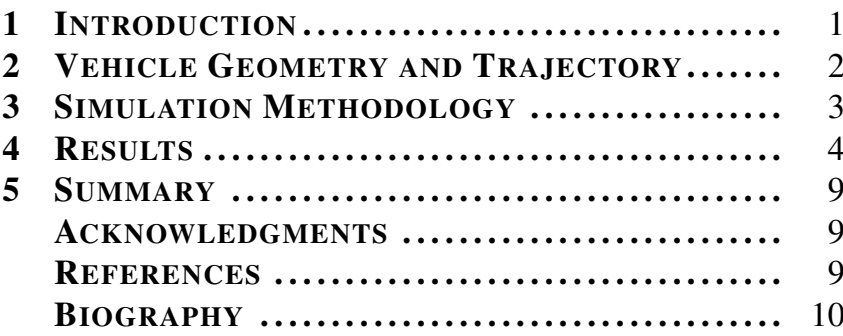

\section{INTRODUCTION}

A mechanically deployable entry system concept, known as the Adaptive Deployable Entry ProjecT (ADEPT) [1], is currently being considered for development at NASA for a future robotic mission to Venus and Saturn, as well as for delivering high-mass payloads to Mars. The mechanically deployable concept is attractive because it allows designers to circumvent the constraint which the launch vehicle fairing typically imposes on the diameter of the aeroshell, by stowing the aeroshell during launch. The ADEPT vehicle will use a flexible thermal protection system (TPS), such

978-1-4577-0557-1/12/\$26.00 (c)2012 IEEE.

${ }^{1}$ IEEEAC Paper \#1690, Version 2, Updated 21/11/2011. as carbon cloth, supported by a system of ribs and struts, which will be deployed - either en route or in orbit - prior to the atmospheric entry phase of the trajectory. By relaxing the constraint on aeroshell diameter, one can achieve much lower ballistic coefficients than are currently possible using existing architectures. Lowering the ballistic coefficient is generally desirable as it allows for trajectories with more benign aerothermal and structural loading. Inability to access these low ballistic coefficients is seen as a primary limiter on future high-mass entry systems, particularly for missions to Mars[2], and thus this concept has the potential for being an enabling technology for future exploration.

Many challenges remain before the mechanically deployable decelerator concept can mature into a viable system. Among them is a thorough understanding of the aerodynamic characteristics and performance of the vehicle. It is known that legacy sphere-cone shaped entry systems, such as that for the Viking lander, can become dynamically unstable in the low supersonic to transonic portion of the trajectory due to the unsteady aerodynamics resulting from the separated wake of the vehicle. In missions to date, the effect of this is usually minimized due to the deployment of either a parachute or, in the case of Mars Science Laboritory (MSL), an active control system in the low supersonic regime. However, current mission architectures for ADEPT call for transonic parachute deployment around Mach 0.8 , therefore it is critically important to understand the aerodynamic behavior prior to the deployment. Furthermore, the nature of this concept introduces possible issues with coupling between fluid and structural modes, which will necessitate a thorough understanding of the fluid mechanics.

The objective of the current study is to investigate and begin to build some understanding of the effect of the wake on the aerodynamic performance of the vehicle using high fidelity modeling and simulation tools, namely the computational fluid dynamics code US3D. For this investigation, a trajectory was generated for ballistic Venus entry of a $70^{\circ}$ sphere-cone configuration, and several points along the trajectory which span the low-supersonic to transonic flow regimes were selected. A notional representation of the vehicle geometry was created, neglecting the ribs and struts, and CFD simulations were run for each of the selected trajectory points. Flow visualizations which yield insight into the dominant fluid mechanics driving the unsteady aerodynamics are presented, as well as time-history, mean, and variance data of relevant 


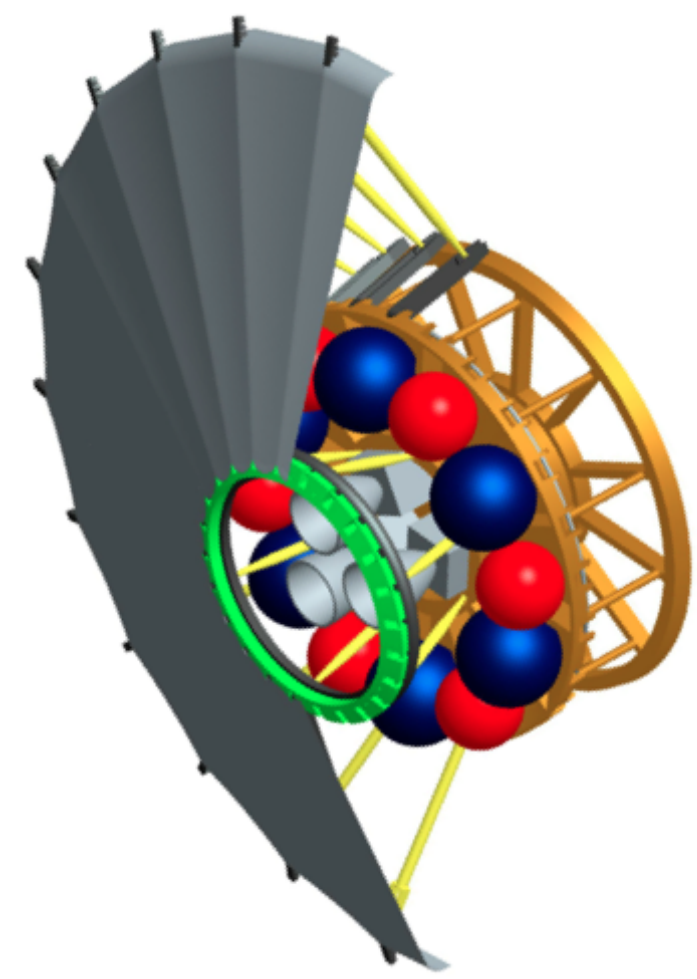

Figure 1. Representative model of a mechanically deployable system. This geometry corresponds to the TEST vehicle [1]

static aerodynamic coefficients. A primary goal of this work is to identify some of the challenges involved in modeling and simulating this flow, and suggest the best simulation methodology going forward. To this end, the effect of various physical modeling choices are examined. In particular, we shall emphasize the differences between using a pure Reynolds Averaged Navier-Stokes (RANS) methodology versus Detached Eddy Simulation (DES) for this type of flow, as well as the effect of using higher order numerical schemes.

\section{VEHICLE GEOMETRY AND TRAJECTORY}

Generating representative boundary conditions for the mission of interest is the first step in simulating the flow. This includes both a realistic representation of the surface geometry, as well inflow conditions and atmospheric composition.

\section{Geometry}

As previously mentioned, the mechanically deployable concept proposes to have a large heat shield made of a flexible TPS material supported by a system of articulated ribs and struts. Figure 1 shows a rendering of one such mechanically deployable vehicle (in this case, the vehicle shown is TEST [1], which is a predecessor to the current ADEPT concept). The nozzles in the nose of the vehicle are for a retropropulsion system which is optional, however these are not simulated for the current work. The geometry simulated in this work is meant to be representative of the vehicle prior to the deployment of any retropropulsion system when there will be a rigid spherical cap on the nose.

This paper will focus on a $70^{\circ}$ cone half-angle configuration

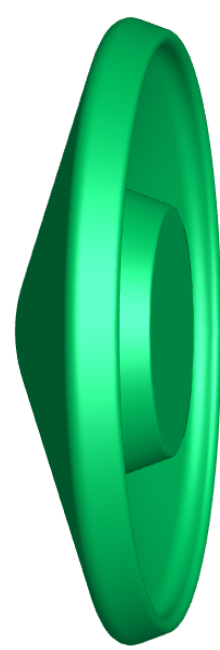

Figure 2. Simplified smooth 70 degree sphere-cone geometry used for all simulations.

of the ADEPT geometry, as this geometry is likely the pointof-departure for future optimization of the design. Also, it is most easily comparable to other legacy sphere-cone geometries, such as those derived from the Viking lander.

For the purposes of this investigation, a simplified representation of this shape has been created and is shown in Figure 2. Note that the sphere-cone forebody of the vehicle is smooth, and does not account for the "sagging" of the fabric. In addition, this model does not include the system of ribs and struts which support the forebody. We expect that the absence of the struts is likely to have a minimal impact as far as the vehicle aerodynamics are concerned. Including them would incur significant cost - both computational and in grid complexity. Finally, it is largely unknown at this stage in the development of the vehicle what the payload might look like due to this likely being mission dependent. For this simplified model, the payload is represented by a short conical frustrum in the center of the base of the vehicle. Examining the sensitivity of the aerodynamics to size and shape of the payload will be a subject for future work.

From a broad fluid mechanical perspective, this geometry differs from the well studied Viking-style family of entry vehicles in that it does not have a conical backshell which tapers smoothly from the shoulder. Instead, there is an abrupt cutoff just aft of the shoulder, and a mostly concave base. Removal of the conical backshell may result in fundamentally different aerodynamic characteristics, due to the different flow physics in the near wake. Additionally, having this type of base likely amplifies some of the errors incurred from employing a pure RANS methodology, as will be shown in the results section.

\section{Trajectory}

A three degree of freedom (3-DoF) simulation of the trajectory for the vehicle entering the Venus atmosphere was performed using the trajectory analysis tool, TRAJ [3]. The input parameters given to TRAJ for the trajectory are shown in Table 1.

TRAJ uses engineering fidelity aerodynamic models and 
Table 1. Input parameters for the 3-DoF trajectory simulation of Venus entry.

\begin{tabular}{|l|c|}
\hline Entry Mass & $2000 \mathrm{~kg}$ \\
Entry Flight Path Angle & $-10^{\circ}$ \\
Entry Velocity & $11.5 \mathrm{~km} / \mathrm{s}$ \\
Ballistic Coefficient & $20 \mathrm{~kg} / \mathrm{m}^{2}$ \\
Capsule Diameter & $8.6 \mathrm{~m}$ \\
\hline
\end{tabular}

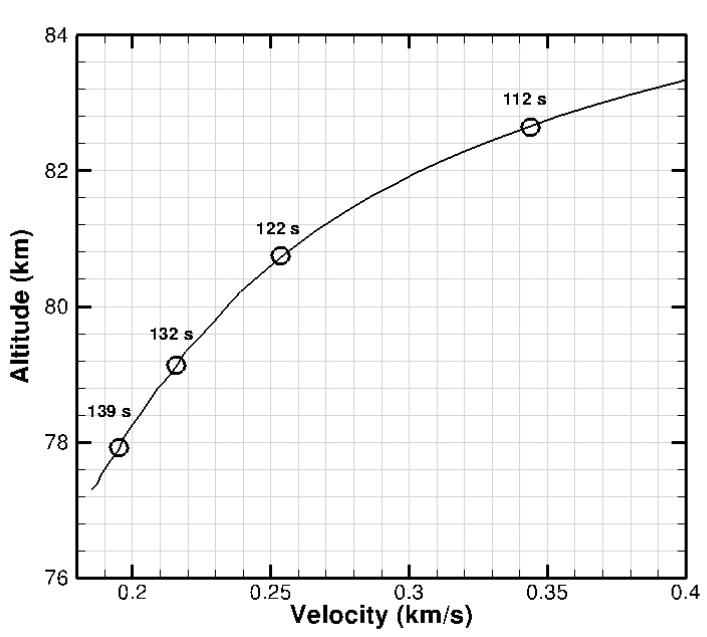

Figure 3. Relevant portion of the trajectory for ballistic Venus entry generated by TRAJ for the present work. The circles indicate the discrete trajectory points selected for simulation. The time after entry is shown above each point in seconds. Note that the entry interface is assumed to be at an altitude of $200 \mathrm{~km}$.

databases, as well as a model of the Venusian atmosphere to generate up to 6-DoF trajectory simulations for entry vehicles. For this simulation, the vehicle's trajectory was constrained to be ballistic $\left(\alpha=0^{\circ}\right)$, and thus restricted to 3 -DoF. It should be noted that the results from the 6 -DoF simulation showed the vehicle to be dynamically unstable below about Mach 1.1. The models for a $70^{\circ}$ sphere-cone in TRAJ are based on Viking-I, whose angle of attack was diverging as an undamped sinusoid at the time of parachute deployment around Mach 1.1, according to flight data. Figure 3 shows the portion of the trajectory output which is of interesest in the present study. Freestream conditions from this trajectory were used to create inflow conditions for the four discrete trajectory points selected for this study. The four points selected are indicated by circles in figure 3 , and the freestream conditions from these points are shown in table 2.

\section{Simulation Methodology}

The following two subsections will briefly describe the simulation setup and numerical methods used to generate the data.

\section{Grid Generation}

When doing simulations of massively separated flows, it is critically important to generate a high quality computational mesh. Poor grid quality can introduce numerical dissipation

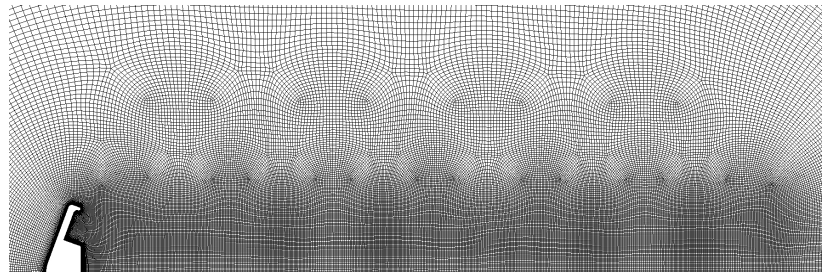

Figure 4. Wake portion of the grid used for all simulations. Note that the grid is refined within the wake while being comparatively coarse outside of the wake region.

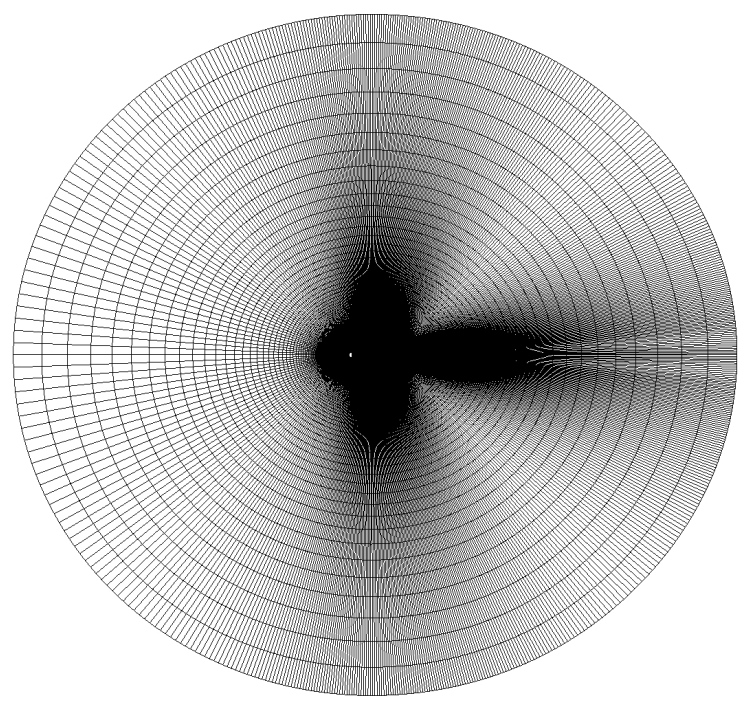

Figure 5. Wide view of the mesh used which shows the exaggerated stretching of the grid near the boundaries. The size of the cells at the boundary are of the order of the diameter of the vehicle.

and drastically affect the accuracy of one's results, as well as adversely affecting numerical stability.

A commercial grid generation package, GridPro [4], was used to generate the grid topology and smooth the body-fitted mesh. Care is taken to maintain local grid isotropy in the wake region, meaning that the computational cells are nearly uniform and cartesian in this area of interest. Furthermore, the topology is designed such that one can cluster points in regions where strong gradients and unsteadiness are likely to be present (in this case, the wake), while having a fairly coarse mesh in more quiescent regions of the flow. This is achieved by using a nested topological refinement strategy which can be seen in the plane shown in Figure 4. Although the vehicle and the forebody flow may be symmetric (or even axisymmetric), the unsteady flow in the vehicle wake will be three dimensional, and therefore for DES calculations the entire flow must be simulated. The portion of the grid shown in this figure represents one half of a symmetry slice of the full three dimensional grid.

The main metric used for sizing the wake of the grid for this study was the number of cells along a diameter of the probe. The final grid used for the simulation had approximately 120 cells across the wake. Finally, as is often done in transonic flow simulations, the refined portion of the grid was extruded to a large distance from the probe resulting in a large sphere as the total computational domain, as depicted in Figure 5. In general, the boundary for this grid is approximately 30 
Table 2. Flight conditions for each trajectory point simulated. (Reynolds number is based on maximum diameter of the vehicle, which for all of this paper is taken to be $8.6 \mathrm{~m}$.)

\begin{tabular}{|l|c|c|c|c|c|c|}
\hline Mach & $U_{\infty}[\mathrm{m} / \mathrm{s}]$ & $\rho_{\infty}\left[\mathrm{kg} / \mathrm{m}^{3} \times 10^{-3}\right]$ & $T_{\infty}[\mathrm{K}]$ & $R e_{D}\left[\times 10^{6}\right]$ & $q_{\infty}[\mathrm{Pa}]$ & $\alpha$ \\
\hline 1.5 & 343 & 0.67 & 187.96 & 2.09 & 394.12 & $0^{\circ}$ \\
1.1 & 259 & 1.18 & 196.98 & 2.64 & 601.40 & $0^{\circ}$ \\
0.9 & 216 & 1.41 & 200.64 & 2.58 & 328.92 & $0^{\circ}$ \\
0.8 & 195 & 1.82 & 205.68 & 2.93 & 346.03 & $0^{\circ}, 0.5^{\circ}, 1^{\circ}, 2^{\circ}, 5^{\circ}$ \\
\hline
\end{tabular}

vehicle diameters from the vehicle itself.

\section{Flow Solver}

The computational fluid dynamics (CFD) solver used for all simulations is US3D. US3D is an unstructured, three dimensional, finite-volume, Navier-Stokes solver developed at the University of Minnesota[5], [6]. The code has multiple time-integration and flux evaluation methods available to the user. It can be run fully laminar, fully turbulent, or tripped to turbulent. In all of the cases presented in this work, implicit time-integration was performed using an implementation of the Full Matrix Data-Parallel Method [7] which is accurate to $2^{\text {nd }}$ order. Most relevant to the present work are the recently added higher order inviscid flux evaluation methods, as well as the choice of turbulence model, so these will be discussed briefly in greater detail.

Higher Order Inviscid Fluxes-Except where otherwise noted, the inviscid portion of the fluxes were computed using the Kinetic Energy Consistent (KEC) scheme of Subbareddy and Candler [8]. This is essentially a hybrid upwindsymmetric scheme which splits the flux into a dissipative (upwind) part and a non-dissipative (symmetric) part. The dissipative part is then added only in the presence of shocks or strong gradients using the switch proposed by Ducros [9]. Using a gradient based extrapolation to the cell faces allows this method to be up to $6^{\text {th }}$ order accurate. Using this method allows for computations which resolve much finer structure than standard upwind methods, while still maintaining numerical stability.

Turbulence Modeling-The selection of an appropriate turbulence model can have a large effect on the accuracy of the results. Often, the Reynolds Averaged Navier-Stokes (RANS) family of turbulence models are used for doing aerodynamic calculations. This approach typically solves a time averaged form of the governing equations, with a closure model to appropriately account for the diffusion of energy due to turbulence, with the expectation that the solution will accurately represent the mean flow. For many problems, particularly ones with attached boundary layers, this approach can be quite effective. However, for massively separated flows, which feature large recirculating unsteady regions, this approach is often inadequate.

An alternative which has become quite popular is Detached Eddy Simulation (DES). The idea behind DES is that fluid motions down to the grid scale are simulated directly, while the turbulent motion which is of a smaller scale than that of the grid cell is modeled. Ideally then, when one constructs a grid for performing a DES, one does so such that the grid resolves a majority of the energy contained in the unsteady fluid motion. This method is then attractive because the requirements it imposes with respect to grid size and therefore computational cost are much less stringent than that for
Large Eddy Simulation (LES) or Direct Numerical Simulation (DNS), but still result in an accurate representation of the large-scale physics in the flow. The implementation of DES employed in the present work is that described by Strelets [10], using the Spalart-Allmaras [11] one equation turbulence model with the Catris-Aupoix [12] compressibility correction to model the turbulent diffusion not resolved by the grid, as well as the turbulent boundary layer.

\section{RESULTS}

The results of the simulations performed for this study will be presented in the ensuing subsections. First, we will briefly describe the Viking wind tunnel data which has been used for comparison. Then, the results of the four ballistic trajectory point simulations are presented. Finally, the Mach 0.8 case is examined in greater detail as a way of evaluating the effect of various numerical modeling choices. Also, several angles of attack are simulated for this case.

\section{Viking Wind Tunnel Data}

As this vehicle concept is still in the early stages of development, there have been no wind tunnel tests to date at these conditions to compare to the computation. Furthermore, there are relatively scant amounts of test data on the transonic aerodynamics of planetary probe shaped entry vehicles in general. However, during the Viking program, a series of wind tunnel tests were done to characterize the aerodynamic performance of the capsule at transonic speeds [13]. Of most relevance to this study were cases run where they tested only the aeroshell without the lander and backshell. Renderings of the two test models can be seen in Figure 6. Note that the model on the right, which represents the aeroshell-only configuration, bears a qualitative resemblance to the notional model of ADEPT being used in this study in that it has a hollow base.

It should be stressed that, while this model does bear qualitative similarities to the geometry in this study, it is not the intent of the authors to suggest that this data represents a validation case for the numerical simulations in this paper. There are significant mismatches in scale, gas chemical composition, and Reynolds number (the wind tunnel tests ranged from about $0.74 \times 10^{6}$ to $\left.1.84 \times 10^{6}\right)$. Rather it is an attempt to anchor the results in the context of historical data, and perhaps draw some qualitative comparisons.

\section{Trajectory Simulation}

Here, we present results of the DES simulations of the four trajectory points spanning the low supersonic to transonic regimes. All of the cases presented in this section were run at $0^{\circ}$ angle of attack (ballistic trajectory) with $6^{\text {th }}$ order accurate inviscid fluxes. Figure 7 shows instantaneous pitch plane slices with contours of constant Mach number. 

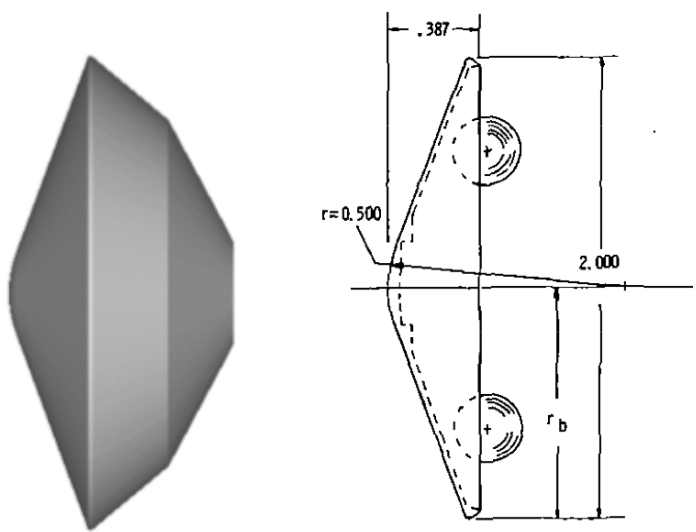

Figure 6. Renderings of the test models used in the Viking transonic wind tunnel tests. The model on the left represents the full entry vehicle, while the drawing on the right is of the aeroshell-only configuration, and was taken directly from ref. [13].

Instantaneous snapshots of the flow as it transverses these flow regimes gives an idea of how the general flow topology evolves over the duration of this portion of the trajectory. Beginning with Mach 1.5, we see the canonical supersonic blunt body type flow features: a fairly strong bow-shock, an abrupt separation and rapid expansion near the shoulder of the vehicle, a subsonic recirculation region in the wake near the back of the vehicle, and a fairly rapid recompression and reattachment some distance downstream of the vehicle. We can also begin to see a fairly coherent "wagging" of the tail of the wake near the right edge of the domain in the figure. At Mach 1.1, we still observe many of the same flow features as in the previous case, however in this case, the reattachment and closure of the wake is much less coherent. Again we can begin to see some "wagging" of the tail of the wake far downstream of the vehicle.

In the transonic regime, at Mach 0.9 and 0.8 , we observe more or less a complete dissociation of the coherent closure of the wake, particularly for the Mach 0.8 case, and there is wide spread unsteadiness far downstream of the vehicle.

By averaging the time history of the data over a representative amount of flow time, we can estimate the drag performance of the vehicle over this range of Mach numbers. The timeaveraged axial force coefficients (for the ballistic trajectory points, this is the drag) based on this calculation are shown in Figure 8 by the red set of points. Also seen in this figure are the drag coefficients from the Viking tests. The green set shows the values for the entire entry vehicle, whereas the blue curve is for the aeroshell-only configuration. We observe that over this range of conditions, the vehicle's drag performance is comparable to that of the Viking entry vehicle.

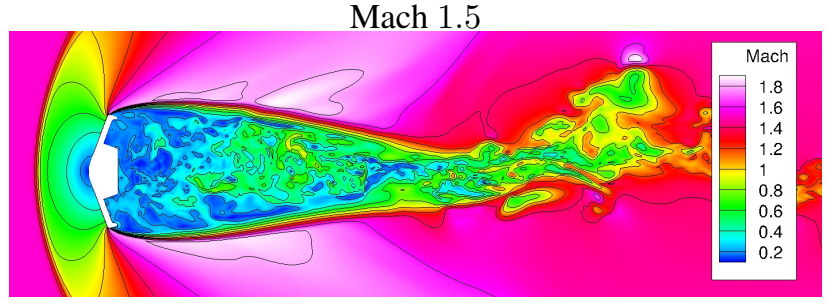

Mach 1.1


Figure 7. Instantaneous pitch plane slices showing contours of constant Mach number for each of the trajectory points simulated..

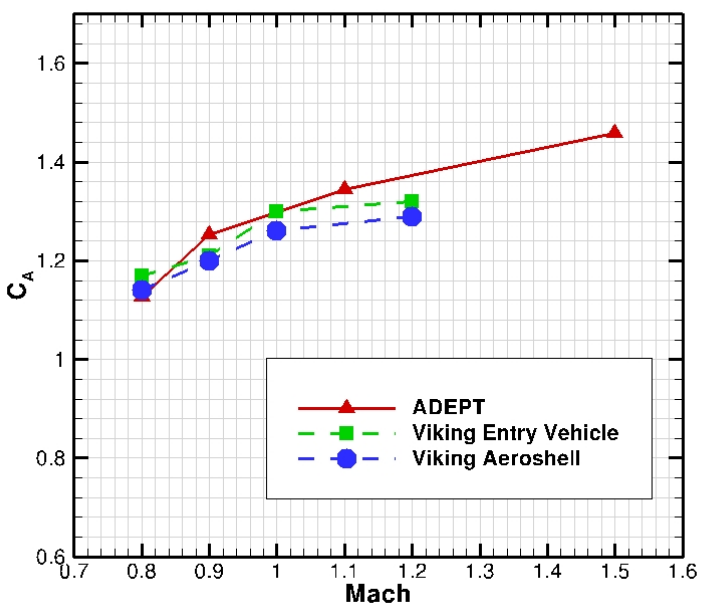

Figure 8. Computed axial force coefficients for the vehicle over the simulated range of Mach numbers. The red triangles are the computed values for the DES simulation, while the green squares are the experimental values for the full Viking entry vehicle, and the blue circles are the experimental values for Viking aeroshell-only configuration. 
Transonic Case Study (Mach 0.8)

Of primary interest to this investigation is to begin to characterize how the unsteady wake affects the static aerodynamic performance of the vehicle. Therefore, we will examine in greater detail, at several angles of attack, the trajectory point at the lowest end of the Mach number range: Mach 0.8. It is assumed that it would be at this point where the effect would be most pronounced as the base pressure, and pressure fluctuations will be non-trivial compared to the pressure on the forebody. As the Mach number increases, the forebody pressure will dominate, and small fluctuations on the backshell are decreasingly likely to have any destabilizing effect. Additionally, in this section, we shall demonstrate the effect of different choices with respect to the numerical method, and the turbulence model.

Higher Order Numerics-As stated at the outset, for the most part, the cases presented in this study were run using a stable, low-dissipation scheme which is spatially $6^{\text {th }}$ order accurate. It is instructive to demonstrate how significant an effect greater spatial accuracy can have on a massively separated flow such as this. To this end, the plots in Figure 9 shows isosurfaces of the so-called Q-criterion (formally, the second invariant of the velocity gradient tensor) [14], which is a tool for visualizing coherent vortical structures in an unsteady flow. The bottom plot in this figure shows results obtained using a $2^{\text {nd }}$ order upwind method, such as that found in many flow solvers in use today. The top plot shows results obtained using the $6^{t h}$ order low dissipation method on the same computational mesh. Qualitatively, we can observe much smaller and finer structure in the top figure. This is further illustrated in figure 10, where we have plotted positive contours of $\mathrm{Q}$ for the less dissipative method, and the upwind method on the top and bottom, respectively. Note the disparity in the length scales in the near-wake in particular.

In the case of this flow, resolving more of the physics in this manner has a strong effect on the wake induced aerodynamic fluctuations. This can be seen in figure 11, which shows representative time histories of the pitching moment coefficient for the $6^{t h}$ order scheme in red, and the upwind scheme in blue. The time on the horizontal axis has been normalized by the diameter of the vehicle divided by the free stream velocity. We note that the highest amplitude fluctuations for the upwind scheme are about two times greater than those for the less dissipative scheme. Furthermore, we can observe that there is much more content at high frequencies, but low amplitudes, in the trace for the less dissipative scheme. In general, by resolving more of the energy spectrum of the wake, we allow the flow to store energy in smaller scale motions. From the perspective of the surface of the vehicle, this results in more smaller fluctuations which are less correlated than if we constrained the resolved flow to have only large energy containing eddies. This is the case with using the lower order upwind scheme, where, even though the scheme has more artificial dissipation, the scale of unsteady fluid motion near the wall tends to be greater just by virtue of a lack of spatial resolution, and thus the likelihood of a destabilizing wake "event" is more likely.

We can gain further insight into the effect of these choices of numerical method by examining the fluctuations in the pressure in the near-wake region. Figure 12 shows rootmean-square (RMS) of the pressure fluctuations normalized by local mean pressure for the upwind scheme in blue, and the $6^{\text {th }}$ order scheme in red. The top plot is taken from data along the centerline, and the bottom figure is for a line parallel to the
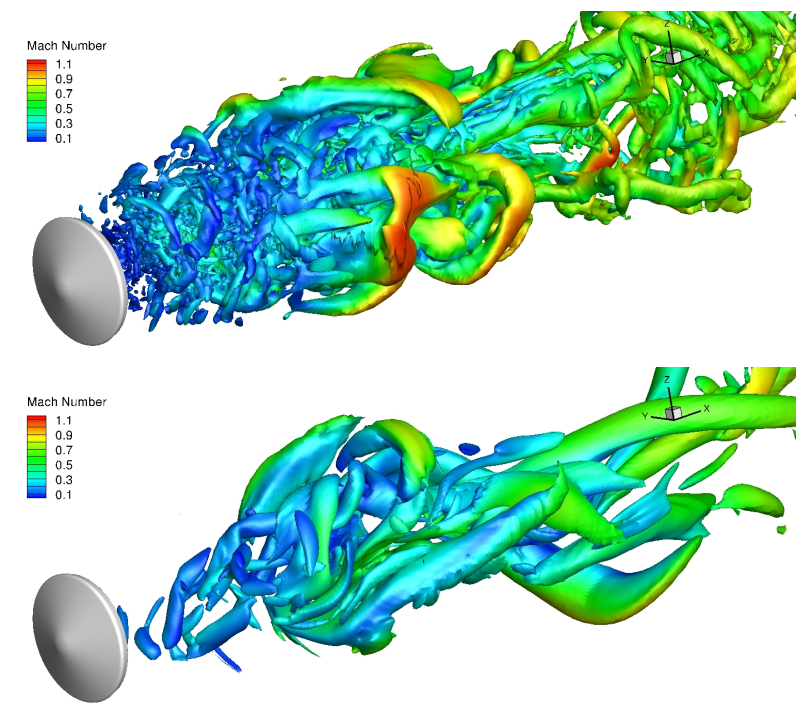

Figure 9. Plots show isosurfaces of the so-called Q-criterion, which is a means for identifying coherent vortical structures in an unsteady flow. The surfaces are colored by the local Mach number.The top figure is of a simulation using the $6^{\text {th }}$ order low-dissipation flux. The figure on the bottom is of result obtained using a typical $2^{\text {nd }}$ order upwind type flux.

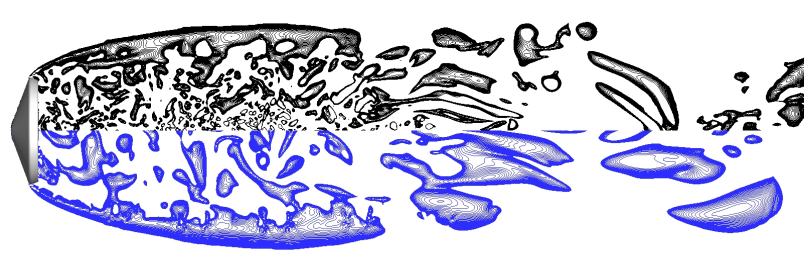

Figure 10. Plot shows contours of positive values of the Qcriterion for both the $6^{\text {th }}$ order KEC method (top), and the $2^{\text {nd }}$ order upwind method (bottom). The positive contours shown here are meant to illustrate the ranges of scales of vortical motion captured by each simulation.

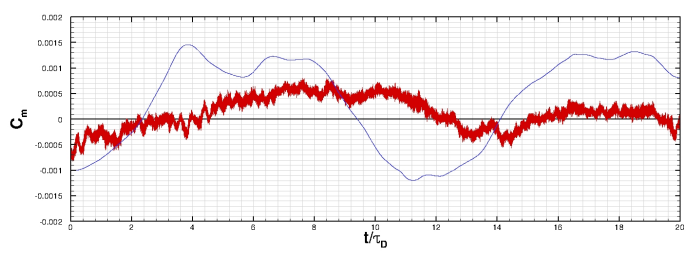

Figure 11. Representative time histories for the pitching moment using the $6^{\text {th }}$ order scheme (red), and the $2^{\text {nd }}$ order upwind scheme (blue) at $\alpha=0^{\circ}$. The time (shown here on the horizontal axis) is normalized by a characteristic flow time based on maximum vehicle diameter and the free stream velocity. 

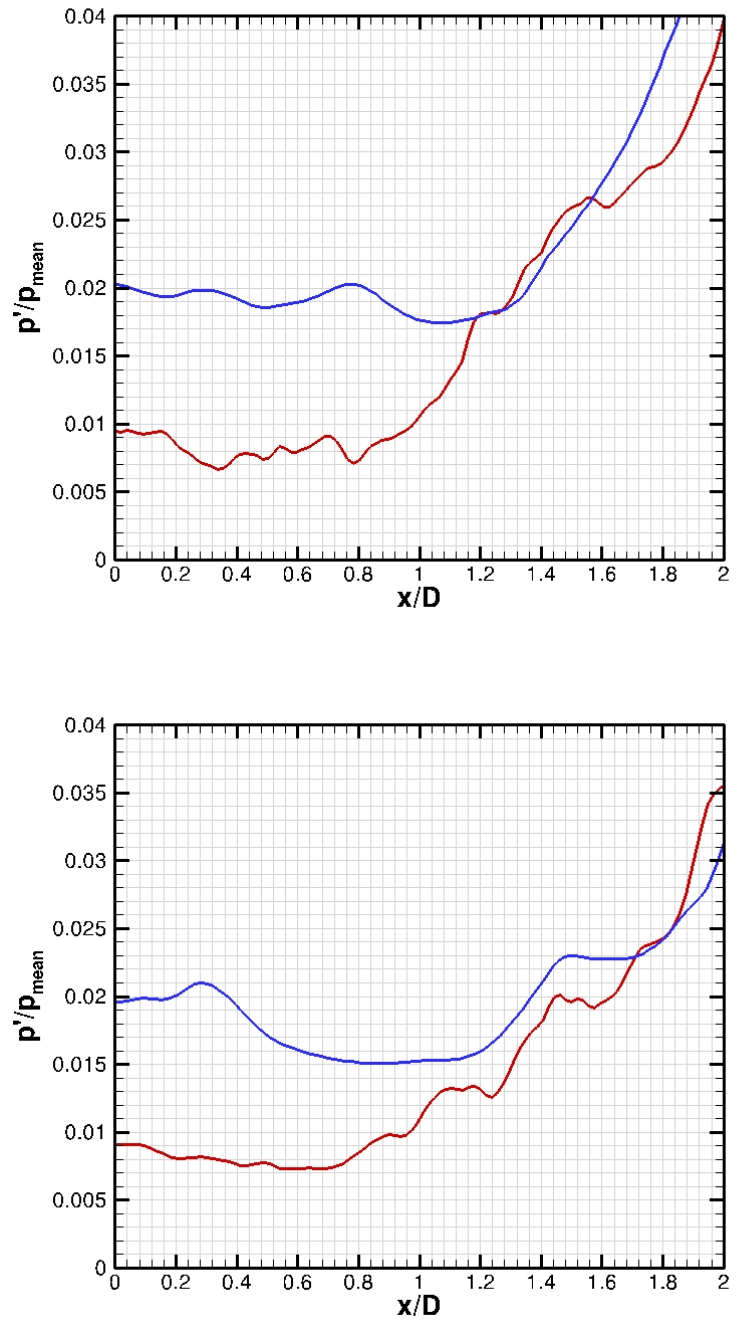

Figure 12. Root-mean-square of the pressure fluctuations normalized by the local mean pressure for both the vehicle centerline (top), and a line parallel to the centerline but 0.25 diameters from the base center point in the radial direction (bottom). The red curve was obtained using the $6^{\text {th }}$ order method, and the blue is using the $2^{\text {nd }}$ order upwind scheme. The horizontal axis gives distance along the line from the vehicle base normalized by the vehicle diameter.

centerline, but through a point 0.25 vehicle diameters from the center axis. The horizontal axis shows the distance from the surface of the base normalized by vehicle diameter. Here we see that in both plots, the amplitude for the higher-order scheme decays more as we move toward the vehicle, with the amplitude near the surface being about half that of the upwind scheme. In figure 13 we compare the mean surface pressure profiles for the two numerical methods, with the upwind method again shown in blue, and the $6^{\text {th }}$ order KEC scheme shown in red. The pressure on the vertical axis has been normalized by the free stream pressure, and the radial position on the horizontal axis has been normalized by the vehicle diameter. The error bars on the data are derived from root-mean-square of the fluctuations in the pressure. Here again, we observe that the band of variation in the pressure for the upwind method is about twice that for the $6^{\text {th }}$ order

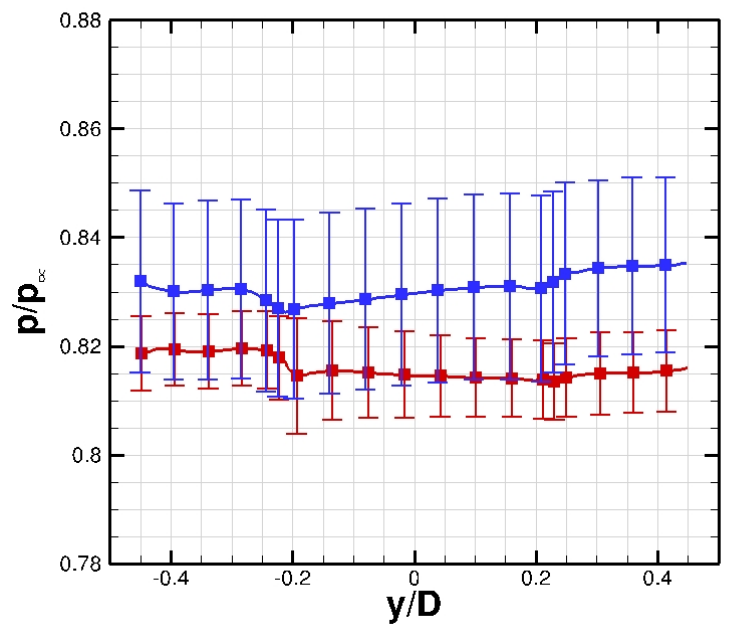

Figure 13. Computed mean base pressure along the surface for $6^{\text {th }}$ order (red), and $2^{\text {nd }}$ order upwind (blue) methods. Error bars represent the root-mean-square of the pressure fluctuations at the surface

scheme. We also note that the mean value for each profile differs slightly, though this difference is likely too small to have a significant influence on the mean aerodynamics.

Static Aerodynamics for DES vs. RANS-In this section we present the computed static aerodynamic coefficients for the capsule at Mach 0.8, and angles of attack between $0^{\circ}$ and $5^{\circ}$. We also computed pure RANS solutions for each of the cases, and the results of these are presented alongside the DES. The goal of RANS modeling is ultimately to accurately represent the mean behavior of the flow through an averaging of the governing equations. Because when using a RANS simulation the mean is essentially computed directly, it achieves statistical convergence much faster than does a calculation which simulates a majority of the unsteady motion of the flow. For this reason, it has some advantages over higher fidelity methodologies in terms of total computational cost, particularly when computing large aerodynamic databases of broad parameter spaces. However for massively separated flows, such as that around this geometry, RANS is does not represent the mean flow well. Thus simulating at least the large unsteady motions directly using higher fidelity - but more costly - techniques, such as DES, is necessary.

The computed axial force coefficients for each angle of attack, shown in red in Figure 14, show striking similarity with those from the Viking wind tunnel data, in particular the coefficients for the aeroshell-only model configuration. The plot also shows how much the results of a pure RANS simulation differ from a DES calculation for this type of flow, even for an integrated quantity. The RANS simulation is represented by black diamonds in this plot and differs from the results of the DES by approximately $20 \%$.

One can account for the discrepancy by looking at the computed mean pressure profiles across the back surface of the vehicle for both RANS and DES. Figure 15 shows such a profile for the cases where $\alpha=0^{\circ}$ (top), and $\alpha=5^{\circ}$ (bottom). In this figure, the mean of the data from the DES is in blue, with error bars based on the RMS of the fluctuations, and the RANS data is in black. We see from the DES a 


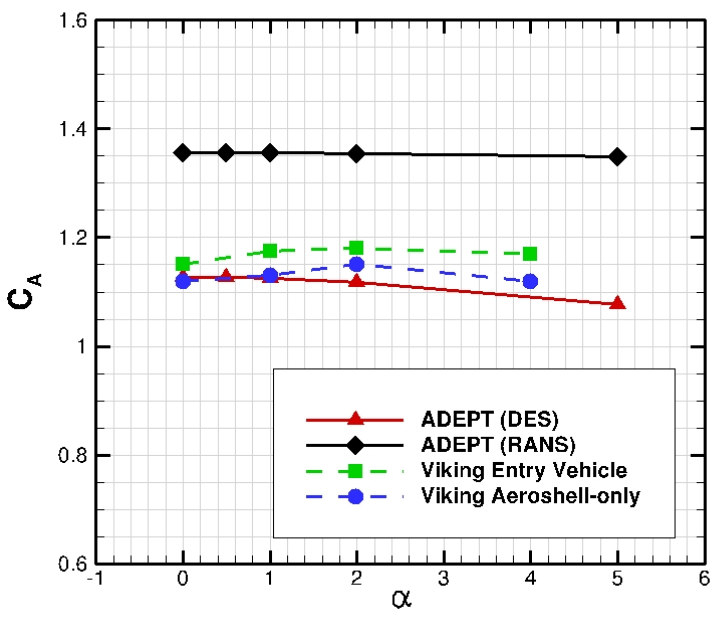

Figure 14. Axial force coefficient versus angle of attack for the DES (red triangles), RANS simulation (black diamonds), and Viking wind tunnel test; both full entry vehicle (green squares), and aeroshell-only (blue circles).

fairly constant mean pressure across the back surface. For RANS, we see a "hump" in the middle of the profile, and an overall much lower pressure than for DES. This discrepancy is very consistent with that observed by Subbareddy [15] in his simulations of supersonic base flow. In that work, through comparison to experiment, the RANS profile was shown to be spurious. A possible explanation for the ability of DES to correctly capture this profile, where the RANS simulation fails, is that the small scale unsteady fluid motions in DES are responsible for rapidly mixing higher temperature gas from the shear layer into the near wake region, thus increasing the static pressure. In contrast, the RANS does not capture such motions, but rather represents the mean in an axisymmetric base flow as a single large toroidal vortex, as seen in figure 16. Furthermore, we observe in this figure that the RANS flow topology essentially results in a jet impinging on the center of the base, which further contributes to the elevated pressure in the center of the RANS profile.

The discrepancy is even more apparent if one compares the mean pressure field in its entirety, as in Figure 17. The top half of this image is the computed mean pressure field from the DES, and the bottom is that from the RANS simulation. Clearly, the mean fields computed by these two methods are quite different. As with the surface profiles, the mixing provided by the small scale unsteady motions of the fluid which are resolved by DES result in a much more uniform pressure field in the wake.

Lastly, in figure 18, we present the computed mean pitching moment coefficient against the angle of attack for the Mach 0.8 case. For the moment coefficient, we have used the projected area based on maximum capsule diameter as the reference area, and maximum capsule diameter as the reference length, as follows:

$$
C_{m}=\frac{M}{q_{\infty} S D} .
$$

Here, $M$ is the moment, $q_{\infty}$ is the free stream dynamic pres-


Figure 15. Surface pressure normalized by the free stream pressure on the vehicle diameter coplanar with the pitch plane, at $\alpha=0^{\circ}$ (top) and $\alpha=5^{\circ}$ (bottom), for both the DES (blue), and the RANS simulation (black). The error bars on the DES curve are based on the RMS of the pressure fluctuations. The outline of the vehicle geometry is shown near the bottom of the figure for reference.

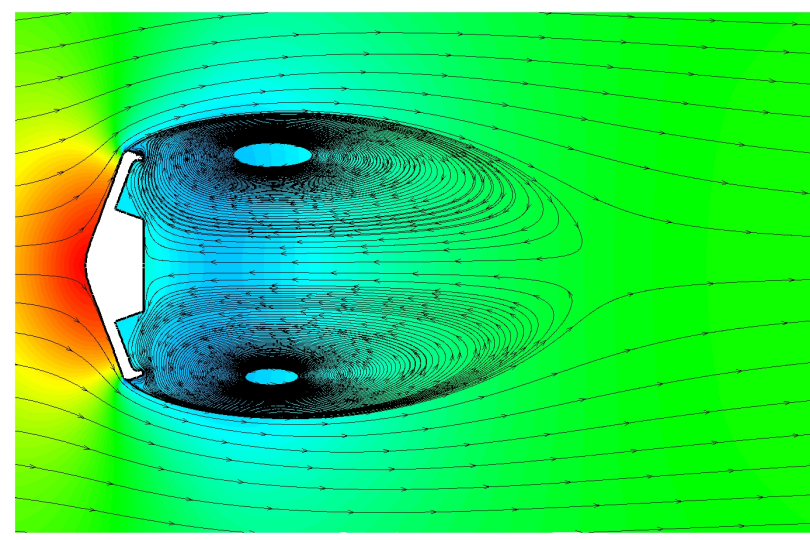

Figure 16. Pitch-plane slice showing computed mean streamlines for the RANS simulation at $\alpha=0^{\circ}$. The contours are of mean pressure. 


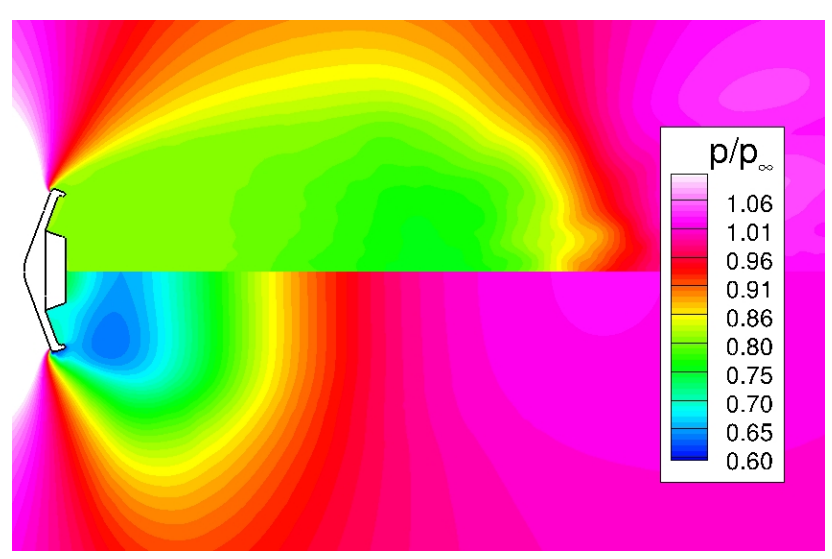

Figure 17. Comparison of the computed mean pressure field in the wake region, normalized by the free stream pressure, for both the DES (top), and the RANS simulation (bottom).

sure, $S$ is the reference area, and $D$ is the maximum capsule diameter. The reference point for the moment was arbitrarily chosen to be the nose of the forebody of the vehicle. In the figure, the DES result is shown in red with error bars based on the standard deviation of the time history of the moment, while the data shown in black is from the RANS simulation. The Viking wind tunnel data is again presented in this figure, with the full entry vehicle shown in green, and the aeroshellonly configuration shown in blue. It should also be noted that the Viking data was transformed so that moment reference point is the same as that for the simulations. In this figure we see that, first of all, there is very little difference between the two Viking datasets, and that, for the most part, both RANS and DES accurately represent the slope. We do observe that the DES seems to diverge somewhat as the angle of attack increases. This may be due to the fact that the grid was aligned with the wake at alpha $=0^{\circ}$, and the DES solution is showing more sensitivity to grid alignment as $\alpha$ is increased. This would imply that the grid requirements for doing DES will be more onerous than for RANS when building an aerodynamic database due to either needing to generate multiple grids, or needing a broader refinement region in the wake to accommodate all of the parameter set. It may be important to note that for this case the moment reference point was chosen to be on the vehicle axis, but had we chosen a CG which was off-axis (and probably more representative of an actual flight vehicle), then the error observed in the computed axial force from the RANS simulations, would have an impact on the moment predictions, and we might see a greater discrepancy between the RANS data and the experiment.

\section{SUMMARY}

A numerical investigation of the supersonic and transonic flow around a mechanically deployable aeroshell has been performed. The simulations demonstrated the effect of using DES versus RANS, as well as the effect of employing higher order numerical schemes. Furthermore, the aerodynamic data from this study showed at least qualitative similarity to the experimental data for the Viking lander.

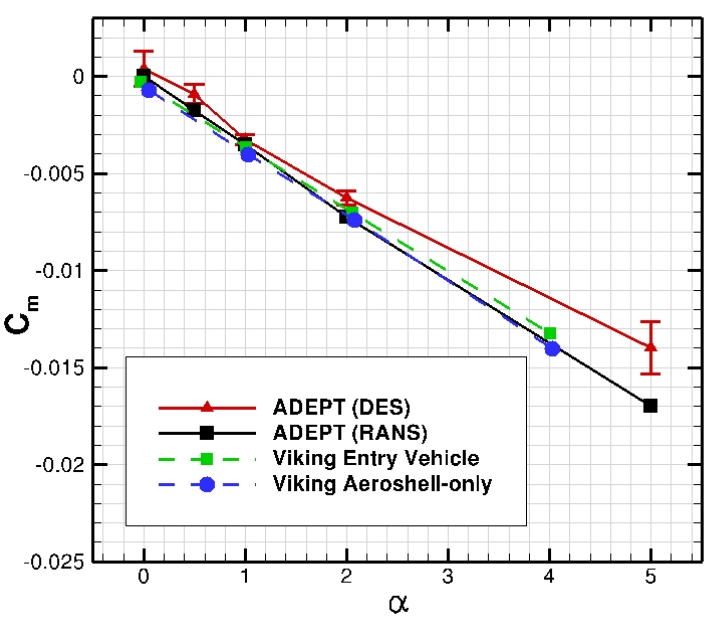

Figure 18. Pitching moment coefficient for the vehicle at Mach 0.8 for both the DES (red triangles), and the RANS simulation (black squares). The Viking wind tunnel data both the full entry vehicle (green), and the aeroshell-only (blue) configurations - has been transformed to have its moment reference center at the nose, to be consistent with the simulation data. The error bars on the DES data are derived from the standard deviation of the time history of the pitching moment.

\section{ACKNOWLEDGMents}

Much of the work in this paper was done at NASA Ames Research Center under the Education Associates Program. Eric Stern would also like to gratefully acknowledge the NASA Office of the Chief Technologist for funding his graduate work through the NASA Space Technology Research Fellowship, under NASA Grant \#NNX11AN42H.

\section{REFERENCES}

[1] E. Venkatapathy, J. Arnold, I. Fernandez, K. Hamm, D. Kinney, B. Laub, M. K. McGuire, K. Peterson, D. Prabhu, D. Empey, I. Dupzyk, L. Huynh, P. Hajela, P. Gage, A. Howard, and D. Andrews, "Adaptive deployable entry and placement technology (adept): A feasibility study for human mission to mars," in $21 s t$ AIAA Aerodynamic Decelerators Systems Technology Conference, no. 2011-2608, Dublin, June 2010.

[2] R. D. Braun and R. D. Manning, "Mars exploration entry, descent and landing challenges," in IEEE Aerospace Conference, December 2005.

[3] G. A. Allen, M. J. Wright, and P. Gage, "The trajectory program (traj): Reference manual and user's guide," NASA, Tech. Rep. TM-2004-212847, 2005.

[4] (2011, December). [Online]. Available: http://www.gridpro.com/

[5] I. Nompelis, T. W. Drayna, and G. V. Candler, "Development of a hybrid unstructured implicit solver for the simulation of reacting flows over complex geometries," in 34th AIAA Fluid Dynamics Conference, no. 20042227, Portland, July 2004.

[6] — "A parallel unstructured implicit solver for hypersonic reacting flow simulation," in 17th AIAA Com- 
putational Fluid Dynamics Conference, no. 2005-4867, Toronto, July 2005.

[7] M. J. Wright, G. V. Candler, and M. Prampolini, "Data parallel lower-upper relaxation method for the navier stokes equations," AIAA Journal, vol. 34, no. 7, pp. 1371-1377, July 1996.

[8] P. K. Subbareddy and G. V. Candler, "A fully discrete, kinetic energy consistent finite-volume scheme for compressible flows," Journal of Comutational Physics, vol. 228, no. 5, pp. 1347-1364, March 2009.

[9] F. Ducros, V. Ferrand, F. Nicoud, C. Weber, D. Darracq, C. Gacherieu, and T. Poinsot, "Large-eddy simulation of the shock/turbulence interaction," Journal of Computational Physics, vol. 152, no. 2, pp. 517 - 549, 1999.

[10] M. Strelets, "Detached eddy simulation of massively separated flows," in 39th AIAA Aerospace Sciences Meeting, no. 2001-0879, Reno, January 2001.

[11] P. R. Spalart and S. R. Allmaras, "A one-equation turbulence model for aerodynamic flows," La Recherche Aerospatiale, vol. 1, no. 1, pp. 5-21, 1994.

[12] S. Catris and B. Aupoix, "Density corrections for turbulence models," Aerospace Science and Technology, vol. 4, no. 1, pp. 1 - 11, 2000.

[13] R. J. McGhee, P. M. Siemers, and R. E. Pelc, "Transonic aerodynamic characteristics of the viking entry and lander configurations," NASA, Tech. Rep. TM-X-2354, September 1971.

[14] J. C. R. Hunt, A. A. Wray, and P. Moin, "Eddies, streams and convergence zones in turbulent flows," in Studying Turbulence Using Numerical Simulation Databases, 2: Proceedings of the 1988 Summer Program, 1988, pp. 193-208.

[15] P. K. Subbareddy and G. V. Candler, "Numerical investigation of supersonic base flows using des," in 43rd AIAA Aerospace Sciences Meeting, no. 2005-886, Reno, January 2005.

\section{BIOGRAPHY}

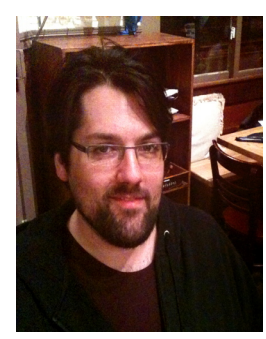

Eric Stern is a graduate student in the department of aerospace engineering and mechanics at the University of Minnesota. His current research focuses on modeling in-depth material response for pyrolyzing ablators. $\mathrm{He}$ is also a recipient of the NASA Space Technology Research Fellowship. He received his Bachelors degree in Aerospace Engineering and Mechanics from the University of Minnesota in 2009.



Michael Barnhardt is a Research Scientist with ERC, Inc., an on-site contractor at NASA Ames Research Center. He received both his Bachelor and Ph.D. in Aerospace Engineering from the University of Minnesota. His research focus is on modeling and simulation of highspeed flow fields, including unsteady wake dynamics, fluid-structure interaction, and material response coupling. He is currently Lead Developer for aerothermal CFD in the Entry Systems and Technology Division at NASA Ames Research Center.

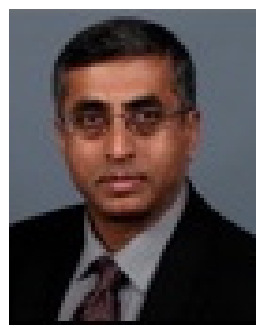

Ethiraj Venkatapathy is currently the Chief Technologist for the Entry Systems and Technology Division at NASA Ames Research Center. He received his B.Tech. in Aeronautical Engineering from the Indian Institute of Technology Madras, India, and his Ph.D. in Aerospace Engineering from the Iowa State University at Ames, Iowa. He is currently leading the technology development efforts, as the PI for the Conformable and Flexible Thermal Protection System (CFA-TPS), the mechanically deployable semi-rigid ADEPT project and Woven Thermal Protection System (WTPS) project.

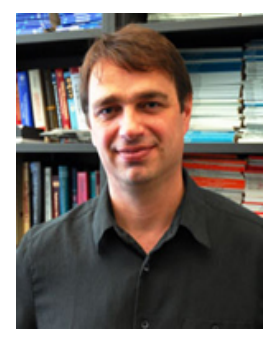

Graham Candler is the McKnight Presidential Professor of Aerospace Engineering and Mechanics at the University of Minnesota. He works on methods for computational fluid dynamics of hypersonic flows and on the development of physical models for high-temperature reacting flows. He did his graduate work at Stanford University and his undergraduate degree is from McGill University. He is a Fellow of the AIAA and holds a National Security Science and Engineering Faculty Fellowship.

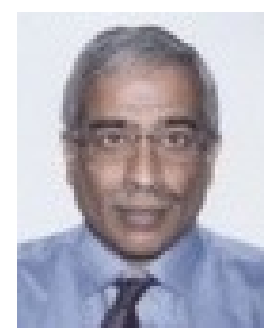

Dinesh Prabhu is a Senior Research Scientist with ERC, Inc., an on-site contractor at NASA Ames Research Center. He received his B.Tech. in Aeronautical Engineering from the Indian Institute of Technology at Madras, India, and his $\mathrm{Ph} . \mathrm{D}$. in Aerospace Engineering from the Iowa State University at Ames, Iowa. His interests are in modeling and simulation of high-temperature hypersonic flow fields, and in aerothermodynamic design of entry vehicles. 